\title{
Sciage De Rebuts De Tectona Grandis L. F. (Teck)
}

\author{
Ahoba Assandé, \\ N'Guessan Kangah Anatole,
}

Centre National de Recherche Agronomique (CNRA)

Station de Recherche Technologique (SRT), Côte d'Ivoire

Gueulou Nina,

Université Nangui Abrogoua,

UFR des Sciences de la Nature (SN), Côte d'Ivoire.

Centre National de Recherche Agronomique (CNRA)

Station de Recherche Technologique (SRT), Côte d'Ivoire

\section{Issali Auguste Emmanuel,}

Ministère de la Recherche Scientifique et de l'Innovation Technologique,

Congo, Brazzaville

\section{Coulibaly Brahima,}

Centre National de Recherche Agronomique (CNRA)

Station de Recherche Technologique (SRT), Côte d'Ivoire

Doi:10.19044/esj.2020.v16n18p140 URL:http://dx.doi.org/10.19044/esj.2020.v16n18p140

\section{Résumé}

Les rebuts de billons de teck abandonnés dans les parcelles après exploitation, sont taxés de faibles diamètres et de faible rendement au sciage. Le travail réalisé vise à montrer la similitude de ces valeurs entre les rebuts et les billons de premier choix. Sept cent (700) rebuts de billons de teck de 24 ans, sélectionnés en zone de forêt dense semi-décidue, ont été cubés et sciés en usine avec un plateau technique adapté aux bois de petits diamètres. Les résultats obtenus montrent que les diamètres moyens des rebuts de billons étudiés sont faibles et gravitent autour de la classe $15 / 19 \mathrm{~cm}$ à $37 \%$, autour de la classe $20 / 24 \mathrm{~cm}$ à $44 \%$ (majoritaire), autour de la classe $25 / 29 \mathrm{~cm}$ à $15 \%$ et autour de la classe 30/34 cm à seulement 3\%. Ces valeurs, bien que faibles, sont du même ordre de grandeur que celles des billons de premier choix de même âge ou d'âge proche. Le rendement brut sciage obtenu est de 38,12\% qui est faible mais également similaire à celui des billons de teck de premier choix. Ce rendement chute de 4 à $7 \%$ en éliminant l'aubier pour améliorer la qualité des débités. Les rebuts de billons de teck présentent des diamètres et des rendements sciages faibles mais similaires à ceux des billons de premier choix. Les quantités abandonnées dans les parcelles prenant de plus en plus de l'ampleur, les rebuts offrent ainsi une bonne opportunité de valorisation de 
matière première à l'échelle industrielle par un plateau technique fixe ou mobile.

Mots clés : Teck, Rebuts, Diamètre, Sciage, Rendement Sciage, Aubier

\title{
Sawing and Sawing Yield of Scrap of Tectona Grandis L. F. (Teak)
}

\author{
Ahoba Assandé, \\ N'Guessan Kangah Anatole, \\ Centre National de Recherche Agronomique (CNRA) \\ Station de Recherche Technologique (SRT), Côte d'Ivoire \\ Gueulou Nina, \\ Université Nangui Abrogoua, \\ UFR des Sciences de la Nature (SN), Côte d'Ivoire. \\ Centre National de Recherche Agronomique (CNRA) \\ Station de Recherche Technologique (SRT), Côte d'Ivoire \\ Issali Auguste Emmanuel, \\ Ministère de la Recherche Scientifique et de l'Innovation Technologique, \\ Congo, Brazzaville \\ Coulibaly Brahima, \\ Centre National de Recherche Agronomique (CNRA) \\ Station de Recherche Technologique (SRT), Côte d'Ivoire
}

\begin{abstract}
Discarded teak logs in post-harvest plots are taxed with small diameters and low sawing yield. The work carried out aims to show the similarity of these values between the rejects and the logs of first choice. Seven hundred rejects of 24-year-old teak logs, selected in semi-deciduous dense forest zone, were cubed and sawed at the factory with a technical platform adapted to small diameter woods. The results obtained show that the average diameters of the discarded teak logs studied are low and revolve around the $15 / 19 \mathrm{~cm}$ class at $37 \%$, around the $20 / 24 \mathrm{~cm}$ class at $44 \%$ (majority), around the $25 / 29 \mathrm{~cm}$ class at $15 \%$ and around the class $30 / 34 \mathrm{~cm}$ at only $3 \%$. These values, although low, are of the same order of magnitude as those of firstgrade logs of the same age or near age.The gross sawing yield obtained is $38.12 \%$ which is low but also similar to that of the first choice teak logs. This
\end{abstract}


yield drops by 4 to $7 \%$ by eliminating the sapwood to improve the quality of the sawn timber. Discarded teak logs have low lumber diameters and yields, but are similar to those of high grade logs. The quantities left in the plots taking more and more scale, the rejects thus offer a good opportunity for raw material valorization on an industrial scale by a fixed or mobile technical platform.

Keywords: Teak, Discarded Logs, Diameter, Sawing, Sawing Yield, Sapwood

\section{Introduction}

Le Tectona grandis (teck) couvre en Côte d'Ivoire 81886 ha, qui correspondent à $31 \%$ des 264139 ha de superficies forestières reconstituées au plan national (SODEFOR, 2012). Il représente 50\% du volume de bois vendu chaque année et constitue la plus grande source d'espoir parmi les six importantes espèces forestières utilisées en reboisement (Tectona grandis, Terminalia superba, Terminalia ivorensis, Cedrela odorata, Triplochiton scleroxylon, Gmelina arborea). En ce qui concerne le Teck, les études récentes ont porté sur l'âge d'exploitabilité technique et la lutte contre les insectes ravageurs (N'guessan et al., 2015 ; Voui Bi et al., 2016).

L'exploitation commerciale des arbres matures de cette essence par des contrats de gré à gré est souvent à l'origine d'énormes gaspillages, par l'abandon sur le terrain d'importantes quantités de bois. Ces quantités sont évaluées pour l'année 2011 à plus de $1600 \mathrm{~m}^{3}$ de rebuts pour la Téné et 550 $\mathrm{m}^{3}$ pour la Sangoué. Les rebuts ainsi produits restent sans acheteur, car font l'objet de nombreuses récriminations en termes de petits diamètres, de faibles rendements, de nombre élevé de nœuds, de mauvaise conformation, de proportion importante d'aubier et de dépréciation de la qualité du bois. Le stockage de ces rebuts inflammables dans les parcelles avec des saisons sèches parfois très prononcées, entraîne non seulement un manque à gagner mais également un danger énorme pour l'avenir des périmètres reboisés en cas d'incendie. Cette situation inquiétante a suscité la mise en œuvre d'un programme de collaboration entre le développement et la recherche pour la détermination de la qualité des rebuts de Teck. Les actions à exécuter englobent entre autres la cotation des billons, le cubage, le sciage, le rendement sciage, le classement des débités, la détermination de la qualité du bois et la confection d'articles de démonstration.

Le présent article traite spécifiquement de l'estimation du diamètre, du sciage et surtout du rendement sciage qui figurent en bonne place des récriminations faites aux rebuts de billons de teck. 


\section{Matériel et méthodes \\ Matériel végétal}

Le matériel végétal est constitué de $53 \mathrm{~m}^{3}$ représentant 700 rebuts de billons de Teck (Tectona grandis L.f) âgés de 24 ans. Ces rebuts ont séjourné plus de 12 à 16 mois en plantation, après exploitation et récupération des meilleures parties du tronc. La figure 1 présente une vue de ce matériel végétal.

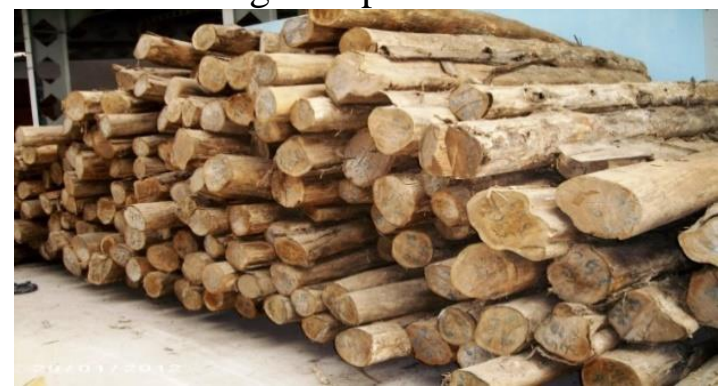

Figure 1 : Rebuts de billons de teck livrés à l'usine

\section{Matériel technique}

Le matériel technique utilisé une unité industrielle de bois créée en 2009, sur un domaine de 2,3 ha situé dans la zone industrielle de Koumassi à Abidjan. La scierie est équipée de deux (2) lignes (LT300) de sciage pour les grumes de gros diamètres et d'une (1) ligne SLP (Small Logs Processing) pour les bois de petits diamètres. Le sciage des rebuts de billons de teck a été réalisé sur la ligne SLP destinée aux billons de petits diamètres. Le chargement du banc de scie et le retournement de la grume sont semi-manuels. Cette ligne à pilotage semi-automatique comporte en tête de sciage, deux scies verticales jumelées à bande étroite (32 - $50 \mathrm{~mm}$ de large) et de marque wood mizer TVS (Twin Vertical Saw). Les scies ont un volant de $600 \mathrm{~mm}$ de diamètre et sont légèrement inclinée par rapport à la verticale. Un système d'arrosage maintient les lames lubrifiées à l'eau pour réduire l'échauffement et accroître ainsi leur performance générale. Dans la technologie adoptée sur cette ligne, la scie de tête est fixe et c'est la grume ou le billon qui se déplace dans une gorge en $\mathrm{V}$ grâce à une chaîne roulante munie de taquet poussoir en fer. La grume est poussée contre les lames par le taquet de la chaîne roulante et deux dosses d'épaisseur désirée sont enlevées simultanément au passage. La grume dépouillée de deux dosses est reprise à l'étape suivante par une scie verticale pour l'enlèvement de la troisième dosse. A la dernière étape, la grume ne comportant qu'une dosse est reprise par une série de trois scies à lame horizontale préréglées aux épaisseurs désirées (Figure 2). Quatre planches sortent simultanément en bout de chaîne. Les sciages obtenus sont acheminés par des chaînes et des roulettes vers une déligneuse multiple munie de deux lames (Figure 3) opérationnelles. Cette déligneuse est équipée de rayons laser permettant de visualiser le passage du trait de scie sur l'aubier à déligner. 


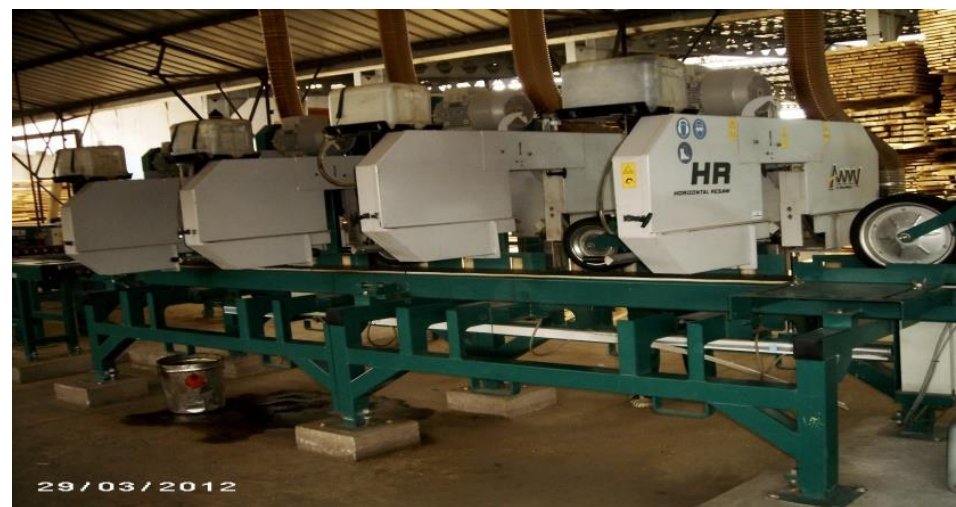

Figure 2: Série de 4 scies dont 3 utilisées dans le cadre de cette étude

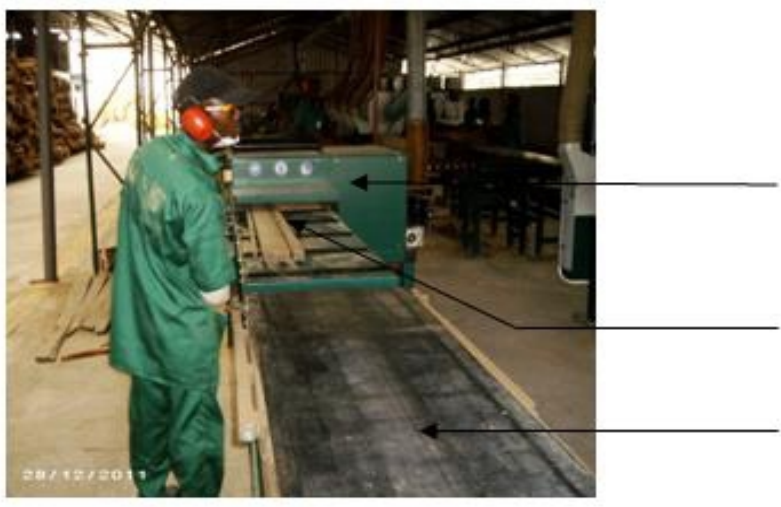

Bâti de la déligneuse

multiple

Planche en délignage

avec deux lames

Tapis de convoyage des

produits délignés

Figure 3: Déligneuse multilames équipée de deux lames

\section{Méthodes}

\section{Echantillonnage des rebuts}

Trois chargements de rebuts de billons de tecks récupérés dans les parcelles de Téné (région d'Oumé en Côte d'Ivoire) par l'Unité de Gestion Forestière (U.G.F) de ladite localité ont été livrés à la scierie Kamaad en zone industrielle de Koumassi pour transformation et évaluation de leur qualité. Cette livraison de $53 \mathrm{~m}^{3}$ composée de 700 billons a été répartie en différents lots pour la conduite de l'étude.

- semi-manuel sur une scie mobile HR 115 d'éléments destinés au marché indien (spécifiés au tableau1dans la suite du document)

- Lot 2 - sélection de 40 billons bien conformés et de gros diamètres pour sciage en chaîne d'éléments de portes

- Lot 3- sélection de 50 billons de moins bonne conformation, non retenus par les deux premiers lots, pour sciage semi-manuel sur une scie mobile HR 115 d'éléments de lamellés-collés (spécifiés au tableau 2 dans la suite du document) 
- Lot 4 - sélection de 20 billons bien conformés et de gros diamètres pour sciage en chaîne d'éléments de portes

- Lot 5 - sélection de 19 billons de moins bonne conformation pour sciage en chaîne d'éléments de lames (spécifiés au tableau 2 dans la suite du document)

- Lot 6 - sélection de 21 billons de moins bonne conformation pour sciage en chaîne d'éléments de lamellés-collés

- Lot 7- sélection de 446 billons regroupés indépendamment de la conformation pour sciage en chaîne d'équarris, de lames et d'éléments de lamellés-collés

\section{Cubage des billons}

Chaque billon numéroté à la réception, a fait l'objet d'un cubage en termes de mesure de diamètres et de longueurs. Pour soixante d'entre eux, le cubage a pris en compte le bois parfait et l'épaisseur d'aubier. Deux mesures de diamètres sous écorce (D1, D2) ont été prises en croix au petit bout et deux mesures (D3, D4) en croix au gros bout. Le bois parfait a également fait l'objet de deux mesures de diamètre (BP1, BP2) en croix au petit bout et de deux mesures de diamètre (BP3, BP4) en croix au gros bout pour les billons retenus. Cette approche a permis de déduire l'épaisseur de l'aubier à chaque extrémité. Les expressions [1], [2] et [3] permettant d'accéder aux valeurs moyennes sont :

$$
\begin{gathered}
\text { Diamètre moyen }\left(\mathrm{D}_{\mathrm{m}}\right) \text { sous écorce du billon }=\frac{\mathrm{D}_{1}+\mathrm{D}_{2}+\mathrm{D}_{3}+\mathrm{D}_{4}}{4} \\
\text { Diamètre moyen du bois parfait }(\mathrm{BP})=\frac{\mathrm{BP}_{1}+\mathrm{BP}_{2}+\mathrm{BP}_{3}+\mathrm{BP}_{4}}{4} \\
\text { Epaisseur moyenne d'aubier }\left(\mathrm{E}_{\mathrm{a}}\right)=\frac{\left(\mathrm{D}_{\mathrm{m}}-\mathrm{BP}\right)}{2}
\end{gathered}
$$

En assimilant chaque billon à un cylindre, le volume du billon $\left(\mathrm{V}_{\mathrm{b}}\right)$ a été calculé selon la formule de Huber (Anonyme, 1989) par l'expression [4]:

$$
\mathrm{V}_{\mathrm{b}}=\frac{\pi\left(\mathrm{D}_{\mathrm{m}}\right)^{2}}{4} \mathrm{~L}
$$

Avec

$\mathrm{V}_{\mathrm{b}}=$ Volume du billon

$\mathrm{L}=$ Longueur du billon

$\mathrm{D}_{\mathrm{m}}=$ Diamètre moyen du billon

Л $=3,14$

De la même manière le volume du bois parfait $\left(\mathrm{V}_{\mathrm{BP}}\right)$ a été déterminé par l'expression [5] : 


$$
\mathrm{V}_{\mathrm{BP}}=\frac{\pi(\mathrm{BP})^{2}}{4} \mathrm{~L}
$$

Avec

$\mathrm{V}_{\mathrm{BP}}=$ Volume du bois parfait

$\mathrm{L} \quad=$ Longueur du billon

$\mathrm{BP}=$ Diamètre moyen du bois parfait

Л $=3,14$

Le volume d'aubier a été obtenu par différence entre le volume du billon et le volume du bois parfait par l'expression [6] :

$$
\mathrm{V}_{\mathrm{a}}=\mathrm{V}_{\mathrm{b}}-\mathrm{V}_{\mathrm{BP}}
$$

Avec

$\mathrm{V}_{\mathrm{a}}=$ Volume de l'aubier

$\mathrm{V}_{\mathrm{b}}=$ Volume du billon

$\mathrm{V}_{\mathrm{BP}}=$ Volume du bois parfait

\section{Sciage et rendement sciage}

Le mode de sciage adopté à l'usine a consisté à l'état frais en l'enlèvement de deux dosses au premier passage du billon par une scie jumelée comme décrit plus haut (paragraphe 1.1.2). La troisième dosse est enlevée à l'étape suivante par une scie verticale (SVS = Single Vertical Saw) de reprise et enfin des planches d'épaisseurs désirées sont extraites par un ensemble de trois scies horizontales montées en série.

Pour le lot 1 (lot indien) et le lot3, les plateaux centraux grossièrement sciés sur trois côtés par l'enlèvement de trois dosses, ont été mis en tas avec les dosses enlevées. Un sciage semi-manuel sur une scie horizontale (HR115) a été appliqué par la suite à l'ensemble de ces plateaux et de leurs dosses, conformément aux spécifications dimensionnelles (Tableau 1) du technicien indien venu de Tanzanie pour la circonstance. Le sciage adopté a consisté en l'enlèvement de débités de différentes dimensions: équarris englobant pour la plupart le cœur, planchettes, chevrons et coursons. Ces débités ont été cubés pour accéder au rendement sciage par lot.

Pour les autres lots, les planches ont été sciées en chaîne conformément aux spécifications d'épaisseur (Tableau 2) fournies par l'atelier de menuiserie de la So Les avivés obtenus ont aussi fait l'objet de mensurations individuelles de longueur, largeur et épaisseur pour la détermination du volume sciage qui, rapporté au volume grume, permet d'accéder au rendement sciage par lot. 
Tableau 1: Spécifications de sciage pour le marché indien (OLAM)

\begin{tabular}{lccc}
\hline Produits & \multicolumn{3}{c}{ Spécifications } \\
\cline { 2 - 4 } & Longueur $(\mathrm{m})$ & Largeur $(\mathrm{mm})$ & Epaisseur $(\mathrm{mm})$ \\
\hline $\begin{array}{l}\text { A- Eléments pour le } \\
\text { marché général }\end{array}$ & 2,10 et plus & 130 et plus & 75 et 100 \\
\hline $\begin{array}{l}\text { B- Eléments pour } \\
\text { fenêtres }\end{array}$ & 0,91 à 1,82 & 75 et plus & 50 et 63,5 \\
\hline B- Eléments pour portes & 2,10 et plus & 75 et plus & 50 et 63,5 \\
\hline $\begin{array}{l}\text { C- Eléments pour } \\
\text { fenêtres }\end{array}$ & 0,91 à 1,82 & 75 et plus & 38 \\
\hline C- Eléments pour portes & 2,10 et plus & 75 et plus & 38 \\
\hline
\end{tabular}

Tableau 2: Spécifications de sciage fournies par l'atelier de menuiserie de la scierie

\begin{tabular}{lccc}
\hline Produits & $\begin{array}{c}\text { Longueurs } \\
(\mathbf{m})\end{array}$ & Largeurs $(\mathbf{m m})$ & $\begin{array}{c}\text { Epaisseurs } \\
(\mathbf{m m})\end{array}$ \\
\hline $\begin{array}{l}\text { Eléments de } \\
\text { lamellés- } \\
\text { collés }\end{array}$ & 2,$2 ; 2,3$ & $\begin{array}{c}50+2 ; 80+2 ; \\
100+2 ; 150+2\end{array}$ & $\mathbf{3 0 + 2 ; 6 0 + 2}$ \\
\hline $\begin{array}{l}\text { Eléments de } \\
\text { portes }\end{array}$ & 2,15 & 200 & $56+2$ \\
\hline $\begin{array}{l}\text { Eléments de } \\
\text { lames }\end{array}$ & $\mathbf{2 , 1 0 ; 1 , 8 0}$ & $\mathbf{1 5 0}$ pour 120 & $\mathbf{3 0}$ pour 25 \\
\hline
\end{tabular}

\section{Analyse des données}

Les analyses effectuées en termes de statistiques descriptives ont été réalisées à l'aide du logiciel Excel 2010. Les valeurs minimales, les valeurs maximales, les moyennes, les coefficients de variation et le mode ont été relevés pour les diamètres des différents lots de billons.

\section{Résultats}

\section{Cubage des rebuts de billons de teck}

Le tableau 3 rassemble les résultats du cubage par lot des rebuts de billons de teck. Il ressort de ce tableau que les diamètres moyens des rebuts de billons de teck sont variables au sein des différents lots constitués. Les coefficients de variation oscillent entre 9 et $20 \%$. Les nombres les plus élevés de billons par lot apparaissent pour les diamètres $19 \mathrm{~cm}$ (lot 1);25 cm (lot 2) ; 18,5 cm (lot 3) ; 31,5 cm (lot 4) ; 22,25 cm (lot 5); 21,25 cm (lot 6) et $18,5 \mathrm{~cm}$ (lot 7). Les lots 2, 4, 5 et 6 renferment ainsi plus de billons intéressants en termes de diamètres pour la transformation. La répartition en classes, des diamètres de l'ensemble des billons, est résumée dans le tableau 4. Les chiffres entre parenthèses dans la dernière colonne correspondent au pourcentage de billons obtenus pour la classe. Les diamètres moyens obtenus pour les rebuts de teck étudiés sont faibles et gravitent à $37 \%$ autour de la classe $15 / 19 \mathrm{~cm}$, à $44 \%$ autour de la classe $20 / 24 \mathrm{~cm}$ et à $15 \%$ autour de la classe $25 / 29 \mathrm{~cm}$. La classe $30 / 34 \mathrm{~cm}$ enregistre environ $3 \%$ des billons. Les 
maxima de diamètres relevés pour ces billons de 24 ans font apparaître des valeurs élevées qui atteignent pour certains lots : $35,5 \mathrm{~cm}$ (lot 7), 36,25cm (lot 5) et $45,75 \mathrm{~cm}$ (lot 4).

Tableau 3 : Cubage par lot des rebuts de billons de teck

\begin{tabular}{lccccccc}
\hline Libellé & Lot 1 & Lot 2 & Lot 3 & Lot 4 & Lot 5 & Lot 6 & Lot 7 \\
\hline $\begin{array}{l}\text { Nombre total de } \\
\text { billons }\end{array}$ & 101 & 40 & 50 & 20 & 19 & 21 & 446 \\
\hline $\begin{array}{l}\text { Longueur moyenne } \\
(\mathrm{m})\end{array}$ & 2,25 & 2,27 & 2,24 & 2,26 & 2,25 & 2,25 & 2,25 \\
\hline $\begin{array}{l}\text { Diamètre gros bout } \\
(\mathrm{cm})\end{array}$ & 20,97 & 28,1 & 20,24 & 29,23 & 26,65 & 27,95 & 21,98 \\
\hline $\begin{array}{l}\text { Diamètre petit bout } \\
(\mathrm{cm})\end{array}$ & 19,07 & 25,4 & 18,36 & 25,04 & 24 & 23,45 & 19,75 \\
\hline $\begin{array}{l}\text { Diamètre moyen } \\
(\mathrm{cm})\end{array}$ & 20,02 & 26,75 & 19,30 & 27,13 & 25,32 & 25,70 & 20,87 \\
\hline $\begin{array}{l}\text { Coefficient de } \\
\text { variation }(\%)\end{array}$ & 11,89 & 9,57 & 13,63 & 20,32 & 17,50 & 11,76 & 15,55 \\
\hline $\begin{array}{l}\text { Mode du diamètre } \\
(\mathrm{cm})\end{array}$ & 19 & 25 & 18,5 & 31,5 & 22,25 & 21,25 & 18,5 \\
\hline $\begin{array}{l}\text { Diamètre minimum } \\
(\mathrm{cm})\end{array}$ & 16 & 22 & 16 & 20,5 & 19,75 & 20,75 & 15 \\
\hline $\begin{array}{l}\text { Diamètre } \\
\text { maximum (cm) }\end{array}$ & 27,25 & 31,5 & 27,5 & 45,75 & 36,25 & 30,75 & 35,5 \\
\hline $\begin{array}{l}\text { Volume grume } \\
\left(\mathrm{m}^{3}\right)\end{array}$ & 7,189 & $\mathbf{5 , 1 5 2}$ & $\mathbf{3 , 3 4 3}$ & $\mathbf{2 , 7 1 6}$ & $\mathbf{2 , 2 1 4}$ & $\mathbf{2 , 4 8 5}$ & $\mathbf{3 5 , 2 4 2}$ \\
\hline
\end{tabular}

Tableau 4: Répartition en classes de diamètres des rebuts de billons de teck

\begin{tabular}{cccc}
\hline $\begin{array}{c}\text { Classes de } \\
\text { diamètres }(\mathrm{cm})\end{array}$ & $\begin{array}{c}\text { Nombre de billons } \\
\text { concernés au gros } \\
\text { bout }\end{array}$ & $\begin{array}{c}\text { Nombre de billons } \\
\text { concernés au petit } \\
\text { bout }\end{array}$ & $\begin{array}{c}\text { Nombre de billons de } \\
\text { diamètre moyen } \\
\text { concernés }\end{array}$ \\
\hline $10-14$ & 0 & 9 & 0 \\
\hline $15-19$ & 177 & 325 & $261(37,4 \%)$ \\
\hline $20-24$ & 337 & 275 & $309(44,3 \%)$ \\
\hline $25-29$ & 134 & 79 & $106(15,2 \%)$ \\
\hline $30-34$ & 36 & 7 & $19(2,7 \%)$ \\
\hline $35-39$ & 12 & 2 & $1(0,1 \%)$ \\
\hline $40-44$ & 0 & 0 & 0 \\
\hline $45-49$ & 0 & 0 & $1(0,1 \%)$ \\
\hline $50-54$ & 1 & 0 & 697 \\
\hline Total & 697 & 697 & 6 \\
\hline
\end{tabular}

\section{Sciage et rendement sciage}

Le sciage adopté a permis l'enlèvement de débités de différentes dimensions conformément aux spécifications de sciage données (marché indien, atelier de menuiserie de la société Kamaad) : équarris englobant la plupart du temps le cœur, planches, planchettes (frises), chevrons et coursons. Le nombre total de débités produits est de 3022 qui se repartissent en : 
- 672 planches $(21 \%)$ de différentes épaisseurs $(25,30,35,40,45,50$, 55 et $60 \mathrm{~mm}$ ) destinées au marché général ;

- 1765 frises (55\%) de différentes épaisseurs $(15,19,22,25,30$ et 35 $\mathrm{mm})$ destinées au marché particulier;

- 526 chevrons (19\%) de différentes largeurs (40 à $150 \mathrm{~mm}$ parfois 180 $\mathrm{mm}$ ) et épaisseurs (40 à $80 \mathrm{~mm}$ parfois $100 \mathrm{~mm}$ ) destinés également au marché particulier.

Les résultats de rendement sciage du tableau 6, montrent des moyennes variant de 27 à $48 \%$. Le rendement élevé de $47,81 \%$ obtenu pour le lot1 (indien) résulte de la récupération intégrale de l'ensemble des dosses exploitables et du traitement en sciage semi-manuel avec des choix appropriés. Ces choix faits en fonction de l'élément à scier ont contribué à accroître le nombre de débités et par conséquent le rendement sciage. Le lot 3 qualifié de teck local et traité de la même manière que le lot 1 par sciage semi-manuel a permis d'obtenir un rendement de 40,58\%. Ce rendement montre que la récupération des dosses n'est pas forcément un gage de rendement élevé au sciage; d'autres facteurs interviennent.

Le lot 2 pour éléments de porte, scié en chaîne, donne un rendement de $27,50 \%$. Ce rendement, le plus faible obtenu au cours du présent travail, s'explique surtout par le sciage fait sans aucune récupération des dosses.

Les autres lots ont tous été sciés en chaîne avec récupération des dosses exploitables et ont donné des rendements de 40 à $43 \%$ à l'exception du lot 5 , prévu pour des éléments de lames, qui a donné un rendement de $30,53 \%$. Ce rendement relativement faible résulte de la conformation des billons de ce lot où les billons présentent un à plusieurs méplats marqués avec de nombreuses traces de nœuds et de bosses.

Le rendement brut global pour l'ensemble des lots étudiés est de $39,83 \%$ avec le volume des déclassés et de $38,12 \%$ sans le volume des déclassés. Ces déclassés correspondent à un rendement de 1,71\% pour les rebuts de teck étudiés.

Le pourcentage d'aubier, préoccupant pour une valorisation en bois d'œuvre, a permis de procéder par calcul, à une élimination quasi totale de l'aubier afin d'apprécier le rendement réel en bois parfait. Les résultats en rendement sciage obtenus par cette investigation sont ceux consignés dans le tableau 7. Ces résultats montrent qu'en procédant à une élimination quasi complète de l'aubier, le rendement global sciage chute à $34,31 \%$. Les écarts observés peuvent varier de 4 à $7 \%$ entre le rendement sciage brut avec aubier et le rendement sciage sans aubier. 
Tableau 6: Rendement global et rendements par lot de sciage de rebuts de teck

\begin{tabular}{|c|c|c|c|c|c|c|}
\hline \multirow[t]{2}{*}{ Désignation } & \multicolumn{2}{|c|}{ Nombre de billons } & \multicolumn{2}{|c|}{ Volume grumes } & \multirow{2}{*}{$\begin{array}{l}\text { Volume } \\
\text { sciage } \\
\left(\mathrm{m}^{3}\right)\end{array}$} & \multirow{2}{*}{$\begin{array}{l}\text { Rendement } \\
\text { sciage }(\%)\end{array}$} \\
\hline & $\begin{array}{c}\text { Total } \\
(\mathrm{u})\end{array}$ & $\begin{array}{l}\text { Echantillon } \\
\text { nés (u) }\end{array}$ & $\begin{array}{c}\text { Total } \\
\left(\mathrm{m}^{3}\right)\end{array}$ & $\begin{array}{l}\text { Echantillon } \\
\text { nées }\left(\mathrm{m}^{3}\right)\end{array}$ & & \\
\hline $\begin{array}{l}\text { Lot } 1 \text { (indien)-sciage } \\
\text { semi-manuel }\end{array}$ & 101 & 97 & 7,189 & 6,932 & 3,314 & 47,81 \\
\hline $\begin{array}{l}\text { Lot 2-sciage en chaîne } \\
\text { d'éléments de portes }\end{array}$ & 40 & 33 & 5,152 & 4,203 & 1,156 & 27,50 \\
\hline $\begin{array}{l}\text { Lot 3-sciage semi- } \\
\text { manuel de lamellés- } \\
\text { collés }\end{array}$ & 50 & 38 & 3,343 & 2,435 & 0,988 & 40,58 \\
\hline $\begin{array}{l}\text { Lot 4-sciage en chaîne } \\
\text { d'éléments de portes }\end{array}$ & 20 & 20 & 2,716 & 2,716 & 1,091 & 40,17 \\
\hline $\begin{array}{l}\text { Lot } 5 \text { - sciage en } \\
\text { chaîne de lames }\end{array}$ & 19 & 19 & 2,214 & 2,214 & 0,676 & 30,53 \\
\hline $\begin{array}{l}\text { Lot 6- sciage en } \\
\text { chaîne de lamellés- } \\
\text { collés }\end{array}$ & 21 & 21 & 2,485 & 2,485 & 1,026 & 41,28 \\
\hline $\begin{array}{l}\text { Lot } 7 \text {-sciage en chaîne } \\
\text { de billons regroupés } \\
\text { pour portes, lames et } \\
\text { lamellés-collés }\end{array}$ & 446 & 446 & 35,242 & 35,242 & $\begin{array}{c}14,98 \\
8\end{array}$ & 42,52 \\
\hline Débités declasses & - & - & - & - & 1,000 & 1,71 \\
\hline Total general & 697 & 674 & 58,341 & 55,775 & $\begin{array}{c}22,23 \\
9\end{array}$ & 38,12 \\
\hline
\end{tabular}

Tableau 7: Rendement sciage de rebuts de teck avec et sans aubier

\begin{tabular}{lcccccc}
\hline Désignation & \multicolumn{2}{c}{ Volume grume $\left(\mathrm{m}^{3}\right)$} & \multicolumn{2}{c}{ Volume sciage $\left(\mathrm{m}^{3}\right)$} & \multicolumn{2}{c}{$\begin{array}{c}\text { Rendement sciage } \\
(\%)\end{array}$} \\
\cline { 2 - 7 } & $\begin{array}{c}\text { Avec } \\
\text { aubier }\end{array}$ & $\begin{array}{c}\text { Sans } \\
\text { aubier }\end{array}$ & $\begin{array}{c}\text { Avec } \\
\text { aubier }\end{array}$ & $\begin{array}{c}\text { Sans } \\
\text { aubier* }\end{array}$ & $\begin{array}{c}\text { Avec } \\
\text { aubier }\end{array}$ & $\begin{array}{c}\text { Sans } \\
\text { aubier* }\end{array}$ \\
\hline $\begin{array}{l}\text { Lot 1 (indien) - sciage } \\
\text { semi-manuel }\end{array}$ & 7,189 & 4,960 & 3,314 & 2,983 & 47,81 & 41,49 \\
\hline $\begin{array}{l}\text { Lot 2-sciage en chaîne } \\
\text { d'éléments de portes }\end{array}$ & 5,152 & 3,555 & 1,156 & 1,040 & 27,50 & 24,74 \\
\hline $\begin{array}{l}\text { Lot 3-sciage semi-manuel } \\
\text { de lamellés-collés }\end{array}$ & 3,343 & 2,307 & 0,988 & 0,889 & 40,58 & 36,51 \\
\hline $\begin{array}{l}\text { Lot 4-sciage en chaîne } \\
\text { d'éléments de portes }\end{array}$ & 2,716 & 1,874 & 1,091 & 0,982 & 40,17 & 36,16 \\
\hline $\begin{array}{l}\text { Lot 5-sciage en chaîne de } \\
\text { lames }\end{array}$ & 2,214 & 1,528 & 0,676 & 0,608 & 30,53 & 27,46 \\
\hline $\begin{array}{l}\text { Lot 6-sciage en chaîne de } \\
\text { lamellés-collés }\end{array}$ & 2,485 & 1,715 & 1,026 & 0,923 & 41,28 & 37,14 \\
\hline $\begin{array}{l}\text { Lot 7-sciage en chaîne de } \\
\text { billons regroupés pour } \\
\text { portes, lames et lamellés- } \\
\text { collés }\end{array}$ & 35,242 & 24,317 & 14,988 & 13,489 & 42,52 & 38,28 \\
\hline \begin{tabular}{l} 
Total general \\
\hline
\end{tabular} & 58,341 & 40,255 & 22,239 & 20,015 & 38,12 & 34,31 \\
\hline
\end{tabular}




\section{Discussion}

La répartition en classes de diamètres des rebuts de teck étudiés, donne des diamètres moyens faibles gravitant majoritairement (44\%) autour de la classe $20 / 24 \mathrm{~cm}$ à 24 ans. Ces valeurs montrent la faible croissance en épaisseur du teck qui, de $18 \mathrm{~mm} /$ an d'augmentation continue sur le diamètre au jeune âge (0-10 ans), se stabilise en moyenne à $8 \mathrm{~mm} / \mathrm{an}$ à partir de 40 ans (Ahoba, 1990). Malgré tout, les potentialités diamétrales sur la base de ces valeurs peuvent avoisiner 30/32 cm à 24 ans au lieu de 20/24 cm obtenus lors de cette étude sur les rebuts. Au regard de ces valeurs les rebuts de teck abandonnés dans les plantations, affichent des valeurs de diamètre nettement plus faibles par rapport à ce qu'elles devraient être. L'explication de l'écart réside dans l'enlèvement des meilleures parties des fûts par les premiers exploitants. Il est toutefois important de souligner que la vigueur de croissance en diamètre pour le teck comme pour la plupart des essences forestières, est fonction de l'indice de fertilité du site. Ainsi, avec un indice de fertilité de 8 pour le site de Téné (région d'Oumé) d'où proviennent les billons étudiés, les diamètres moyens des fûts de teck sont de $21 \mathrm{~cm}$ à 11 ans et de $24 \mathrm{~cm}$ à 17 ans (Dupuy, 1986; Durand, 1985). Il résulte de ces valeurs moyennes, qu'indépendamment des conditions de croissance des arbres, les rebuts étudiés ont de faibles diamètres par rapport à leur âge. Ces valeurs, bien que faibles, sont du même ordre de grandeur que celles des billons de premier choix de même âge ou d'âge proche. Ainsi, les maxima de diamètres relevés dans certains lots de notre étude, atteignent des valeurs élevées de 36 à $45 \mathrm{~cm}$ qui rivalisent avec celles des billons de premier choix. Pour le site de Bamoro (région de Bouaké) d'indice de fertilité 6-7, les diamètres moyens des fûts de teck sont de $19 \mathrm{~cm}$ à $15-21$ ans, de $24 \mathrm{~cm}$ à $25-33$ ans et de $30 \mathrm{~cm}$ à $33-45$ ans (Dupuy, 1986 ; Durand, 1985). Des recherches effectuées sur des tecks de 50 ans de Bamoro montrent que le diamètre moyen obtenu sur 75 individus est de $35,6 \mathrm{~cm}$ avec un minimum de $27,75 \mathrm{~cm}$ et un maximum de $52,25 \mathrm{~cm}$ (Espinet, 1980).

L'essence n'atteint son plein diamètre d'exploitabilité $(60 \mathrm{~cm})$ qu'à partir de 75 ans (Ahoba, 1990). A titre indicatif, une essence à croissance rapide comme le cedrela, augmente d'environ $2 \mathrm{~cm} /$ an en diamètre continu (Ahoba, 1989) et peut par conséquent, atteindre $48 \mathrm{~cm}$ à 24 ans et $120 \mathrm{~cm}$ à 60 ans.

Les rebuts de teck de 24 ans donnent avec un plateau technique adapté aux petits diamètres, un rendement brut sciage de 38,12\% (Ahoba et Edi, 2012). Ce rendement est grossièrement similaire à celui obtenu sur des tecks de 30 ans (36\%), par scie mobile circulaire (Ahoba et al., 1995 b ; Ahoba et Edi, $1995 \mathrm{c}$;) et à celui obtenu sur des tecks de 41 ans (34\%) par scie fixe ordinaire à ruban (Sitbon, 1981). Sur des tecks de 50 ans, des rendements un peu plus élevés $(44,3 \%)$ ont pu être obtenus par scie fixe ordinaire à ruban 
(Espinet, 1980). D'une manière générale, la tendance qui transparaît est que le rendement sciage des rebuts de billons de teck est du même ordre de grandeur que celui obtenu avec les essences de plantation (Durand, 1981, 1983, 1984 ; Gasnier, 1981). Avec un plateau technique approprié, les rebuts de teck peuvent être valorisés avec un rendement sciage similaire aux tecks de premier choix et essences ordinaires de plantation. Les pourcentages de déclassés obtenus $(1,7 \%)$ peuvent être considérés comme très faibles comparativement à des essences comme le Gmelina arborea et le Pterocarpus erinaceus (vêne) qui donnent respectivement des pourcentages de déclassés de 8,3\% et de 7\% (Ahoba et Edi, 1993 ; Ahoba et al., 1995 a). En procédant à une élimination quasi complète de l'aubier pour améliorer la qualité des débités, le rendement brut sciage chute de $38,12 \%$ à $34,31 \%$ avec des pertes qui peuvent être considérées comme acceptables. L'option valorisation en bois d'œuvre avec réduction importante de l'aubier est ainsi envisageable avec les rebuts de teck.

\section{Conclusion}

Les rebuts de billons de teck présentent de faibles diamètres gravitant majoritairement autour de la classe $20-24 \mathrm{~cm}$. Ces faibles valeurs sont du même ordre de grandeur que celles des billons de premier choix et permettent de les transformer en bois d'œuvre par un plateau technique adapté aux petits diamètres. Cette transformation conduit à un rendement sciage intéressant du même ordre de grandeur que ceux des tecks de premier choix et même des essences ordinaires de plantation. L'élimination quasi complète de l'aubier pour améliorer la qualité des débités conduit à une baisse de 4 à $7 \%$ de ce rendement sciage. La présente étude permet de recommander la valorisation à l'échelle industrielle des rebuts de Tecks par un plateau technique fixe ou mobile.

\section{References :}

1. Ahoba A. (1989). Etude de la qualité du bois de cedrela à partir des cernes d'accroissement annuels. Rapport scientifique interne CTFTCôte d'Ivoire, $20 \mathrm{p}$.

2. Ahoba A. (1990). Etude de la qualité du bois de Teck à partir des cernes d'accroissement annuels ( $1^{\text {ère }}$ partie). Rapport scientifique interne CTFT- Côte d'Ivoire, $10 \mathrm{p}$.

3. Ahoba A. et Edi K. (1993). Rendement sciage, classement et séchage du Gmelina arborea. Rapport scientifique interne CTFT-Côte d'Ivoire, $13 \mathrm{p}$.

4. Ahoba A., Edi K. \& Coulibaly K. (1995 a). Le Pterocarpus erinaceus - Rendement et classementsciage. Rapport scientifique interne IDEFOR/DFO-Côte d'Ivoire, 8 p. 
5. Ahoba, A., Parant B. \& Daigremont C. (1995 b). Valorisation des bois d'éclaircies de Teck et de Gmelina provenant des plantations de la région de Bouaké au moyen de la scie mobileCTFT/ERVE. CIRAD/IDEFOR-DFO, $56 \mathrm{p}$.

6. Ahoba, A. \& Edi, K. (1995 c). Première appréciation de la scie mobile en Côte d'Ivoire - Application au sciage mobile de bois d'œuvre de Teck et de Gmelina à Bennafoko-Centre de gestion Sodefor de Bouaké. Rapport scientifique interne IDEFOR/DFO-Côte d'Ivoire, 29

7. Ahoba, A. \& Edi, K. (2012). Sciage et rendement sciage de rebuts de Tectona grandis L.F. (Teck) Rapport scientifique interne CNRA Côte d'Ivoire, 52p.

8. Anonyme, (1989). Mémento du forestier «Techniques rurales; en

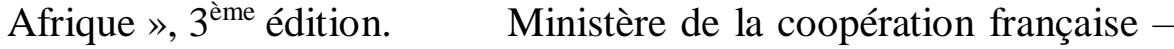
Centre Technique Forestier Tropical, 1266 p.

9. Dupuy, B. (1986). Principales règles de sylviculture pour les plantations à vocation bois d'œuvre. Rapport scientifique interne CTFT Côte d'Ivoire. $112 \mathrm{p}$.

10. Durand, P. Y. (1981). Etude du sciage du Teck provenant de la plantation villageoise de Kasumbarga (Korhogo). Rapport scientifique interne CTFT, Abidjan, Côte d'Ivoire, 16 p.

11. Durand, P. Y. (1983). Production de lattes à partir de Teck d'éclaircie en vue d'une production de panneaux d'ébénisterie en bois massif reconstitué. Rapport scientifique interne CTFT Côte d'Ivoire, $17 \mathrm{p}$.

12. Durand, P. Y. (1984). La technologie du teck en Côte d'Ivoire. Rapport scientifique interne CTFT Côte d'Ivoire, $56 \mathrm{p}$.

13. Durand, P. Y. (1985). Quelques résultats d'essais technologiques concernant les principales essences de reboisement de Côte d'Ivoire. Rapport scientifique interne CTFT Côte d'Ivoire, $21 \mathrm{p}$.

14. Espinet, B. (1980). Etude du sciage du teck : Règles de classement des sciages-Evaluation du rendement matière. Rapport scientifique interne CTFT Côte d'Ivoire, $50 \mathrm{p}$.

15. Gasnier, J. L., (1981). Etude du sciage du Gmelina (Bamoro 1948 et 1953). Rapport scientifique interne CTFT Côte d'Ivoire, 8 p.

16. N'guessan K. A. Voui Bi B. N. B., Traoré S. \& Tapé-Bi F. A. (2015). Productivité maximale et âge d'exploitabilité technique de Tectona grandis (teck) en fonction de la fertilité des stations en Côte d'ivoire, Journal of Applied Biosciences 95:9003 - 9014

17. Sitbon, R. (1981). Etude du sciage de trois (3) lots de teck. Rapport scientifique interne CTFT Côte d'Ivoire, $10 \mathrm{p}$. 
18. SODEFOR (2012). Ensemble des reboisements gérés par la SODEFOR. Document interne de travail/Direction Technique / Sous-Direction Reboisement, $4 \mathrm{p}$.

19. Voui Bi B. N. B, N'guessan K. A., Kassi K.F.J.M., Tapé-Bi F. A \& KAMANZI K. (2016). Insectes ravageurs et champignons parasites associés au dépérissement des peuplements de

Tectona grandis (teck) régénérés à Téné, zone semi-décidue de Côte d'Ivoire. Int. J.Biol. Chem. Sci. 10(1): 87-105. DOI : http://dx.doi.org/10.4314/ijbcs.v10i1.7 